\title{
Profile of temsirolimus in the treatment of advanced renal cell carcinoma
}

This article was published in the following Dove Press journal:

OncoTargets and Therapy

18 September 2010

Number of times this article has been viewed

\author{
Michael Staehler \\ Nicolas Haseke \\ Wael Khoder \\ Christian G Stief \\ Department of Urology, University \\ of Munich, Klinikum Grosshadern, \\ Germany
}

\begin{abstract}
Temsirolimus is a potent inhibtor of the mammalian target of rapamycin (mTOR). In various clinical trials temsirolimus has shown an overall survival benefit for patients with metastatic renal cell carcinoma (mRCC). Thus it is approved for first-line therapy in high-risk mRCC patients. We discuss the indication, side effects and clinical implications of temsirolimus treatment.
\end{abstract}

Keywords: renal cell cancer, nonclear cell renal cancer, temsirolimus, first-line therapy, overall survival, nephrectomy

\section{Introduction}

Renal cell carcinoma (RCC) is the most common malignancy of the functional renal tissue, except the renal urothelium. RCC has an increasing incidence - an estimated 209,000 patients are being diagnosed with cancer of the kidney and renal pelvis per year worldwide, and of these, 102,000 will die. Only $62 \%$ of all RCC patients survive 5 years in all stages. ${ }^{1}$

According to the Heidelberg classification, RCC is divided into several distinct histological subtypes of different tumor entities. Most common are clear cell carcinomas ( $70 \%$ to $80 \%)$, papillary RCC (10\% to $15 \%)$, divided into type I and type II papillary RCC, chromophobe ( $2 \%$ to $4 \%$ ) carcinomas of the collecting ducts of Bellini (CDC) $(0.6 \%$ to $1.2 \%)$ and unclassified RCC.

At initial presentation approximately $30 \%$ of the patients do have synchronous metastatic spread of the disease (mRCC). Another $40 \%$ will develop metachronous metastatic spread. ${ }^{1}$

$\mathrm{RCC}$ is highly vascularized, thus angiogenic therapy has shown high efficacy in the treatment of this disease. The multi-tyrosine-kinase inhibitors (TKIs) sunitinib and sorafenib, the mammalian target of rapamycin (mTOR) inhibitors temsirolimus and everolimus, and the VEGF-antagonist bevacizumab in combination with interferon alpha are available for systemic treatment of $\mathrm{mRCC}$.

With angiogenic treatment based on sunitinib, sorafenib and temsirolimus, progression-free survival (PFS) was mostly doubled, and overall survival (OS) seemed to be prolonged. ${ }^{2-5}$ Thus, angiogenic treatment is regarded as standard therapy in metastatic patients without further surgical treatment choices.

\section{mTOR inhibition}

mTOR, a $250 \mathrm{kD}$ protein kinase, has a key role in regulating translation of transcripts within the processes of angiogenesis and cell growth. Especially, hypoxia-inducible 
factor (HIF) alpha subunits are regulated by mTOR. Regulated by the PTEN/PI3-AKT pathway, mTOR also induces cyclin D1 and cMyc. mTOR inhibition has a direct cytostatic effect by blocking cellular progression from the G1 to the $\mathrm{S}$ phase.

Temsirolimus is a propyl ester analog of sirolimus, also known as rapamycin, a macrolide with antifungal and antitumor properties. Like the parent compound sirolimus, temsirolimus inhibits mTOR. Sirolimus is known as an oral immune-suppressor in transplant patients and has a well known pharmacologic profile. Temsirolimus iv is approved for first-line oncologic patients with poor risk classification, as shown in Table 1.

\section{Study results with temsirolimus}

In a single-agent, phase II study, temsirolimus administration to heavily pretreated patients with $\mathrm{mRCC}(\mathrm{n}=111)$ resulted in a median overall survival of 15.0 months. ${ }^{6}$ Retrospectively, 49 patients were categorized in a poor-risk group according to Memorial Sloan Kettering Cancer Center (MSKCC) risk criteria. ${ }^{7}$ The temsirolimus-treated patients in this group had a 1.7-fold longer median OS than the first-line, interferon alpha (IFN)-treated, poor-risk group reported by Motzer et al. ${ }^{6,7}$ Thus, a phase III study in first-line, poor-risk mRCC patients was initiated in July 2003.

In this large multicenter phase III trial temsirolimus was tested in 626 patients with previously untreated mRCC. In a three-arm study, the combination of temsirolimus (15 mg iv weekly) and IFN (6 million U) was tested against temsirolimus (25 mg iv weekly) and IFN (18 million U) alone. The primary endpoint of a prolonged OS was reached, with patients who received temsirolimus alone having a longer OS (hazard ratio for death, $0.73 ; 95 \%$ confidence interval $[\mathrm{CI}]$, 0.58 to $0.92 ; P=0.008)$ and $\operatorname{PFS}(P<0.001)$ than patients who received IFN alone. OS in the combination-therapy

Table I Risk factors for the stratification of high-risk patients according to the modified criteria used as inclusion criteria for high-risk patients with temsirolimus treatment ${ }^{4}$ disease-free interval of less than a year

- Karnofsky performance index between $60 \%$ to $70 \%$

- hemoglobin level below the lower level of normal

- corrected calcium of more than $10 \mathrm{mg} / \mathrm{dL}(2.5 \mathrm{mmol} / \mathrm{L})$

- lactate-dehydrogenase (LDH) level of more than I.5-fold the upper level of normal

- more than one organ system with metastasic lesions

Note: Three of the six criteria have to be fulfilled to classify patients suitable for temsirolimus therapy. group did not differ significantly from that in the IFN group. Median OS in the IFN group, the temsirolimus group, and the combination-therapy group were $7.3,10.9$, and 8.4 months, respectively. ${ }^{4}$ Although statistically not significant, even the combination of a dose-reduced temsirolimus therapy was beneficial in terms of improved OS than IFN alone.

Patients included had to be high-risk patients by modified MSKCC risk factor as defined by Motzer et al. ${ }^{8}$ Three of 6 criteria had to be fulfilled for the inclusion of patients: a disease-free interval (DFI) of less than a year (time between initial diagnosis and metastatic spread), a Karnofsky performance status of $60 \%$ to $70 \%$, a hemoglobin level below the lower level of normal, a corrected calcium of more than $10 \mathrm{mg} / \mathrm{dL}(2.5 \mathrm{mmol} / \mathrm{L})$, a lactate-dehydrogenase (LDH) level of more than 1.5-fold the upper level of normal and more than one organ system with metastatic lesions (see Table 1). Three of these 6 parameters have to be fulfilled to classify a patient in the high-risk group suitable for temsirolimus therapy.

The IFN-only group received IFN at a starting dose of 3 million $U$ given subcutaneously 3 times per week for the first week. The dose was raised to 9 million U 3 times per week for the second week and to 18 million U 3 times per week for week 3 , if this dose was tolerated. Thus the dose density was limited in the majority of the patients.

\section{Nephrectomy}

A subgroup analysis of the phase III trial was performed to estimate the implication of nephrectomy status in temsirolimustreated patients. Of the 626 patients, 419 (67\%) underwent nephrectomy in the course of their disease prior to study inclusion. The results of this analysis presented at the 2008 ASCO Annual Meeting showed a difference in OS of 10.4 with nephrectomy vs 11.5 months without nephrectomy, but the difference did not reach statistical significance $(P=0.2) .{ }^{9}$ With IFN treatment a slight benefit for OS was seen, with nephrectomy patients surviving 7.8 months after nephrectomy vs 6.2 months without nephrectomy. But again the difference was statistically insignificant $(P=0.47)$.

It remains unclear whether the effect is cause by the nephrectomy itself or by the patient selection, as risk stratification for randomization of the patients was done according to consideration of nephrectomy status. Additionally, there is a lack of information about whether the nephrectomized patients were included with metachronous or synchronous metatastic disease. A Cox regression analysis to identify the factors that might contribute to this effect is still pending. Although proven for non-poor risk patients, in poor-prognosis patients, 
nephrectomy eventually might not be essential to improve OS but prospective trials are pending. ${ }^{10-12}$ Thus the decision to perform debulking nephrectomy in synchronous metastatic poor-risk patients cannot be based on these results.

\section{Clinical implications of temsirolimus treatment}

Given the fact that angiogenic therapy suppresses tumor growth, we think therapy should be performed on a continuous basis until death. Even progression should not lead to a premature end of therapy, as rebound phenomena are commonly described. As temsirolimus is one of several available angiogenic drugs, major clinical problems are the control of its specific side effects, the criteria to determine the efficacy and the right indication for temsirolimus therapy.

\section{Indication for temsirolimus}

The OS benefit was shown in patients who were included based on a modified MSKCC risk classification, as shown in Tables 1 and 2. The difference between the risk classification systems derives mainly from the performance status and the number of organs harboring metastatic lesions. The "temsirolimus" criteria have not yet been validated and in all available risk profiles the number of metastatic organ systems has never been correlated with survival. Thus these criteria have to be interpreted thoroughly for selection of patients. As survival data for patients stratified according to the MSKCC criteria differ significantly, these so-called Motzer criteria are widely accepted in daily clinical practice. The difference between the Motzer criteria and the risk classification of the phase III temsirolimus trial is that in the temsirolimus trial the patients had to have a Karnofsky performance status between $60 \%$ and $70 \%$ and the amount of organs with metastatic lesions was included.

Reclassifying the temsirolimus high-risk patients into the MSKCC risk systems identifies 64 of 209 patients to be

Table 2 Risk factors for the stratification of high-risk patients according to the Memorial Sloan Kettering Cancer Centre (MSKCC) risk classification ${ }^{8}$

- disease-free interval (DFI) of less than a year

- Karnofsky performance status less than $80 \%$

- hemoglobin level below the lower level of normal

- corrected calcium of more than $10 \mathrm{mg} / \mathrm{dL}(2.5 \mathrm{mmol} / \mathrm{L})$

- lactate-dehydrogenase (LDH) level of more than I.5-fold the upper level of normal
MSKCC intermediate-risk compared to 13 of 209 patients being intermediate-risk within the original study criteria.

In the MSKCC intermediate group the OS with IFNonly therapy was 17.7 months compared to 13.0 months with temsirolimus treatment. In the MSKCC high-risk group OS was 6.0 months with IFN only and 10.2 months with temsirolimus. The difference was highly significant $(P=0.0014)$.

Therefore temsirolimus should be regarded a first-line standard in high-risk patients as identified by the 2004 MSKCC risk criteria, as shown in Table 2.

\section{Indication in nonclear cell RCC}

Approximately $80 \%$ of patients had clear cell RCC and $20 \%$ of patients had other histologies, the majority of which were papillary. Patients with clear cell and other RCC histologies, treated with temsirolimus, demonstrated comparable median OS and PFS superior to those treated with IFN alone. Median OS was 10.9 to 13.9 months in nonclear cell carcinomas with a PFS of 3.8 to 7.9 months related to the type of nonclear cell carcinoma. For patients treated with temsirolimus, 59\% with clear cell and $68 \%$ with other RCC histologies experienced tumor reductions compared to $35 \%$ with clear cell and $14 \%$ with other RCC histologies having tumor reductions with IFN alone. ${ }^{13}$ Although sunitinib has shown some efficacy with a median PFS of 5.7 months in papillary RCC in a phase II trial, temsirolimus is the only drug with a analysis of patients with nonclear cell features in a phase III trial. ${ }^{14}$ As a result of these phase III data temsirolimus remains the most efficacious drug in non-clear cell RCC histologies and might be regarded first choice in this subgroup of patients (NCCN guidelines).

\section{Side effects and clinical handling}

In the phase III study the most frequently occurring adverse events greater than grade three were asthenia, anemia and dyspnea. Other important side effects, although mostly grade 2 or less, were rash, stomatitis, diarrhea, vomiting and peripheral edema in about $20 \%$ to $30 \%$ of the cases. Hematologic toxicities and laboratory abnormalities were mainly grade 2 and included hyperlipidemia, hyperglycemia, hypercholesteremia, creatinine increase, thrombocytopenia and, mainly with combination treatment, neutropenia.

Rash, peripheral edema, hyperglycemia and hyperlipidemia were more common in the temsirolimus group, whereas asthenia was more common in the interferon group. There were fewer patients with serious adverse events in the temsirolimus group than in the IFN group $(P=0.02){ }^{4}$ 
In daily practice, in more than 100 patients at our institution hyperlipidemia and thrombocytopenia are mostly clinically unimportant. Elevated creatinine levels might be related to peripheral edema and consecutive hypervolemic hypohydration. Thus diuretics should be started. Also hydrocortisone might be helpful to close capillary leaks and prevent a peripheral edema. In some cases even aggressive diuretic therapy might not overcome the situation. In this case therapy should be paused until re-compensation of the situation. Peripheral and pulmonary edema might lead to weight gain and dyspnea. Treatment should be done with diuretics and/ or hydrocortisone accordingly.

Hyperglycemia might be a problem if clinically symptomatic. $\mathrm{HbA}_{1 \mathrm{c}}$ values should be controlled regularly and if elevation persists with accompanying hyperglycemia anti-diabetes therapy should be started. The effect mainly is reversible when temsirolimus therapy is withdrawn.

Stomatitis and mucositis might not be easily diagnosed, as evident clinical signs are mainly lacking. Thus the patients often complain about pain without any recognizable lesion in the mouth. We have tried several mouth-rinsing lotions with or without local anesthetics but the best relief from the symptoms is achieved by sucking frozen pineapple pieces.

Therapy for rash is unnecessary in most patients. If needed, high-dose topical cortisone should be administered and might be combined with an antibiotic. Topical application of urea was not useful in our patients as it causes itching in most without relief of the rash.

A major problem for a minority of patients is fatigue. We have tried several treatments (such as testosterone, cortisone, physical exercise), but none was helpful. If the patient is really suffering from fatigue, it is important to talk to the relatives. Most partners of ill patients asked them to relax and to avoid any physical exercise. In doing so, fatigue is even intensified.

In a number of patients pneumonitis might be a problem. We have found several patients to show signs and symptoms of this infectious disease. The problem is that the pneumonitis might be regarded as progressive disease by some radiologists, thus resulting in a therapeutic switch. As shown in Figure 1, pneumonitis might show nodular changes, which easily can be misinterpreted as new metastatic lesions. Usually we treat patients with suspected pneumonitis with gyrase inhibitors (mainly moxifloxacin) with continued temsirolimus treatment. Only in very severe cases is systemic mRCC therapy withdrawn after confirmation of the diagnosis by bronchoscopy and pulmonary lavage.

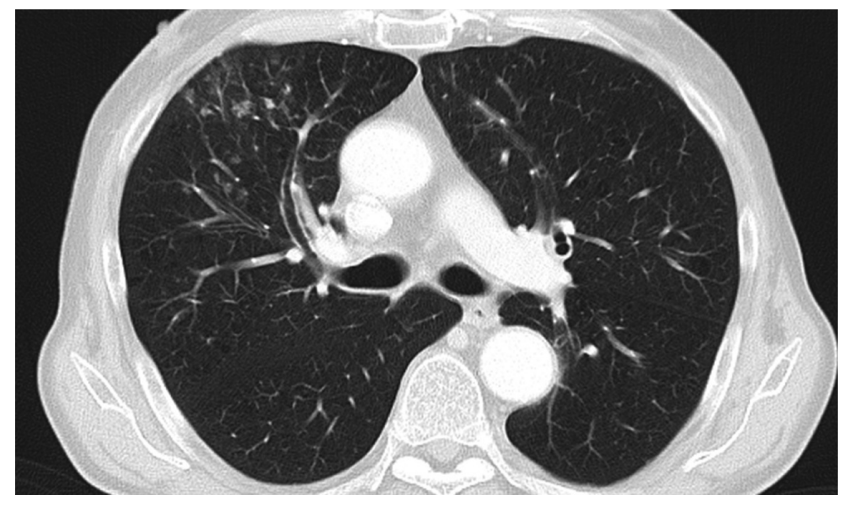

Figure I Right-sided, ventral pneumonitis with temsirolimus treatment resembling new metastastic lesions. After 10 days of antibiotic treatment the lesions faded away completely.

Most side effects are controllable and so far we have not needed to stop therapy due to side effects in any patient.

\section{Evaluation of efficacy}

PTEN and HIF1 alpha have been proposed as predictive biomarkers for temsirolimus treatment. In a substudy of the phase III protocol the baseline status of these molecules was determined and correlated to the clinical outcome of the patients. Unfortunately these levels did not correlate with efficacy in RCC patients treated with temsirolimus. Patients demonstrated OS and PFS benefit when treated with temsirolimus regardless of PTEN and HIF1 alpha status. ${ }^{15}$

In daily treatment serum- or tissue-biomarkers are not used routinely. Therefore it might be beneficial to use information on tumor reassessments, mainly computed tomography (CT) scans, as biomarkers. It has been shown that angiogenic drugs reduce the blood flow in metastatic lesions within hours of administration, resulting in significant reduction in uptake of contrast media into lesions. This can be easily seen on the photographs of contrast media. Even quantification of the blood flow in these lesions is possible with modern radiological scanners (Figure 2). Further investigation is needed to identify the changes in predictive biomarkers. But we already rely on the density of contrast media seen in the metastases on regular CT-printouts to determine the effect of the administered drug. As soon as the lesions gain perfusion compared to the lowest perfusion status achieved (low contrast media uptake), we consider switching the drug.

\section{Discussion}

Because of the recent approval of several drugs for the treatment of $\mathrm{mRCC}$, several criteria must be considered when choosing the best drug for first-line therapy for the 


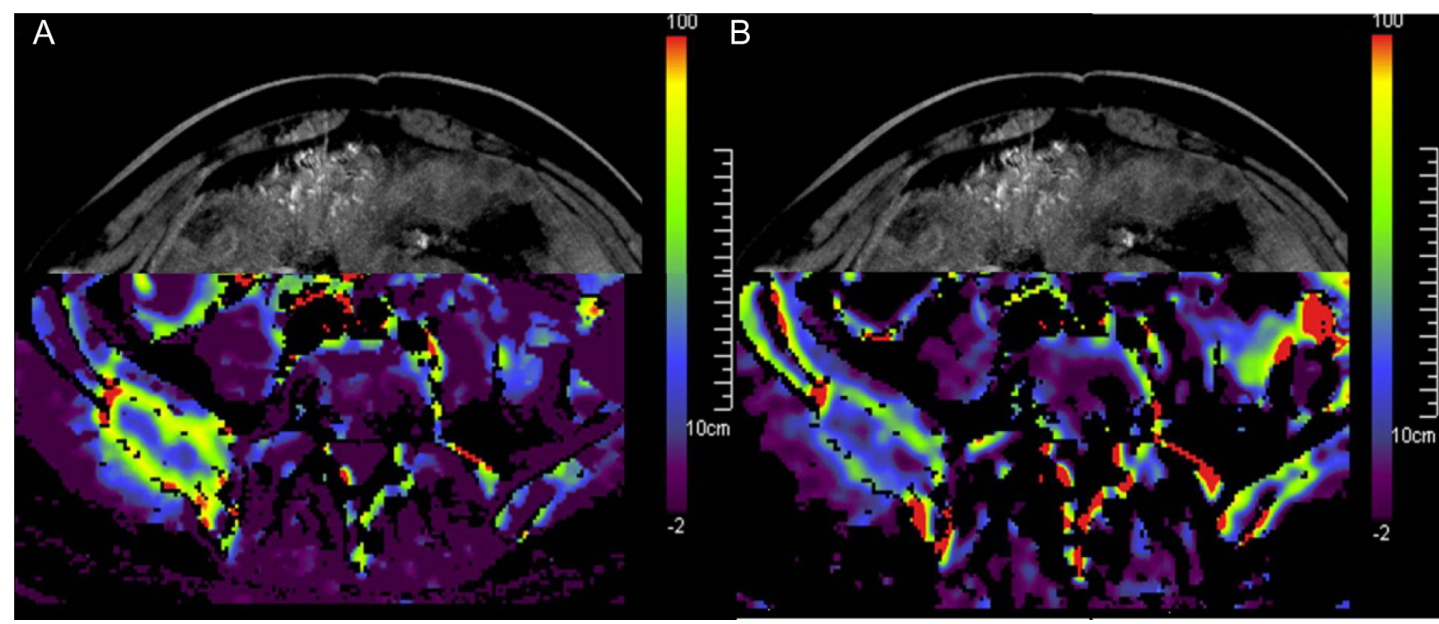

Figure 2 Metastasis of the os ilium: blood flow as quantified by perfusion index measured with computed tomography A) prior to angiogenic therapy; B) same lesion under angiogenic therapy (red: high blood flow, blue: low blood flow). Decreased perfusion in the metastasis caused by angiogenic therapy.

individual patient. We know that most patients will be treated with sequenced therapeutic regimes based on several available drugs and drug classes. ${ }^{16}$ There is now evidence that administering targeted agents sequentially provides clinical benefit by inducing tumor shrinkage and prolonged PFS in a large number of patients. However, data on OS are still pending and will no longer be achievable in controlled trials. With many patients experiencing an OS of more than 40 months in sequenced therapeutic approaches, the situation of $\mathrm{mRCC}$ patients has dramatically improved.

The challenges of the future will be to identify the right patient for the right sequence. Based on several subgroup analyses, temsirolimus seems to provide its best results in patients younger than 65 years, with nonclear-cell histology and a MSKCC high-risk profile in non-nephrectomized patients.

Unfortunately no multi-variate analyses exist to reveal the best prognostic criteria to identify patients who will benefit from temsirolimus therapy in other settings.

Given the fact that a simple modification of the criteria to stratify patients as high risk versus intermediate risk, hints towards the possibility of finding even more sophisticated ways to identify the right patient for an efficacious temsirolimus treatment.

The clinical performance status seems to have a major impact on the effect of temsirolimus, as seen in the MSKCC criteria. But these criteria have been generated in patients who were treated mainly with chemotherapy and immunotherapy based on IFN. The identification of predictive risk factors for patients treated with angiogenic therapy is still pending. Surely not only clinical parameters but histological features should be considered.
We have found a subgroup of patients with good prognosis features who were initially tyrosine kinase inhibitor refractory but sensitive to temsirolimus therapy (data not shown). Future evaluation of first-line patients needs to discriminate between patients who are TKI-resistant but mTOR responders and those who are TKI responders. Also further studies should be undertaken to clarify the role of temsirolimus after TKI therapy.

\section{Conclusion}

Temsirolimus is the only drug for the treatment of mRCC that has a proven OS benefit over IFN alpha in high-risk patients. Thus in this group of patients temsirolimus is recommended as first-line standard. Side effects are controllable. Sequencing TKI therapy and temsirolimus should be evaluated in further protocols. There are suggestions that primary TKI-refractory patients might be treated effectively with temsirolimus. Further efforts should be undertaken to identify mTOR-sensitive and TKI-refractory patients prior to the beginning of first-line treatment.

\section{Disclosure}

The authors declare no conflicts of interest.

\section{References}

1. SEER Cancer Statistics Review, 1975-2005, National Cancer Institute Bethesda, MD, http://seer.cancer.gov/csr/1975_2005/, based on November 2007 SEER data submission, posted to the SEER web site [database on the Internet] 2008.

2. Escudier B, Eisen T, Stadler WM, Szczylik C, Oudard S, Siebels M, et al. Sorafenib in advanced clear-cell renal-cell carcinoma. $N$ Engl J Med. 2007;356(2):125-134.

3. Motzer RJ, Hutson TE, Tomczak P, Michaelson MD, Bukowski RM, Rixe O, et al. Sunitinib versus interferon alfa in metastatic renal-cell carcinoma. N Engl J Med. 2007;356(2):115-124. 
4. Hudes G, Carducci M, Tomczak P, Dutcher J, Figlin R, Kapoor A, et al. Temsirolimus, interferon alfa, or both for advanced renal-cell carcinoma. N Engl J Med. 2007;356(22):2271-2281.

5. Escudier B, Pluzanska A, Koralewski P, Ravaud A, Bracarda S, Szczylik C, et al. Bevacizumab plus interferon alfa-2a for treatment of metastatic renal cell carcinoma: a randomised, double-blind phase III trial. Lancet. 2007;370(9605):2103-2111.

6. Atkins MB, Hidalgo M, Stadler WM, Logan TF, Dutcher JP, Hudes GR, et al. Randomized phase II study of multiple dose levels of CCI-779, a novel mammalian target of rapamycin kinase inhibitor, in patients with advanced refractory renal cell carcinoma. J Clin Oncol. 2004;22(5): 909-918.

7. Motzer RJ, Bacik J, Mazumdar M. Prognostic factors for survival of patients with stage IV renal cell carcinoma: memorial sloan-kettering cancer center experience. Clin Cancer Res. 2004;10(18 Pt 2): 6302S-6303S.

8. Motzer RJ, Bacik J, Schwartz LH, Reuter V, Russo P, Marion S, et al. Prognostic factors for survival in previously treated patients with metastatic renal cell carcinoma. J Clin Oncol. 2004;22(3): 454-463.

9. Logan T, McDermott DF, Dutcher JP, et al. Exploratory analysis of the influence of nephrectomy status on temsirolimus efficacy in patients with advanced renal cell carcinoma and poor-risk features. J Clin Oncol. 2008;26:(May 20 suppl):abstr 5050.
10. Flanigan RC, Mickisch G, Sylvester R, Tangen C, Van Poppel H, Crawford ED. Cytoreductive nephrectomy in patients with metastatic renal cancer: a combined analysis. J Urol. 2004;171(3):1071-1076.

11. Mickisch GH, Garin A, van Poppel H, de Prijck L, Sylvester R. Radical nephrectomy plus interferon-alfa-based immunotherapy compared with interferon alfa alone in metastatic renal-cell carcinoma: a randomised trial. Lancet. 2001;358(9286):966-960.

12. Motzer RJ, Mazumdar M, Bacik J, Berg W, Amsterdam A, Ferrara J. Survival and prognostic stratification of 670 patients with advanced renal cell carcinoma. J Clin Oncol. 1999;17(8):2530-2540.

13. Dutcher JP, de Souza P, McDermott D, Figlin RA, Berkenblit A, Thiele A, et al. Effect of temsirolimus versus interferon-alpha on outcome of patients with advanced renal cell carcinoma of different tumor histologies. Med Oncol. 2009;26(2):202-209.

14. Dutcher JP, de Souza P, Figlin R, et al. Effect of temsirolimus versus interferon- $\alpha$ on survival of patients with advanced renal cell carcinoma of different tumor histologies. Clin Oncol. 2008;26:(May 20 suppl):abstr 384.

15. Figlin RA, de Souza P, McDermott D, Dutcher JP, Berkenblit A, Thiele A, et al. Analysis of PTEN and HIF-1alpha and correlation with efficacy in patients with advanced renal cell carcinoma treated with temsirolimus versus interferon-alpha. Cancer. 2009;(16)3651-3660.

16. Escudier B, Goupil MG, Massard C, Fizazi K. Sequential therapy in renal cell carcinoma. Cancer. 2009;115(10 Suppl):2321-2326.
OncoTargets and Therapy

\section{Publish your work in this journal}

OncoTargets and Therapy is an international, peer-reviewed, open access journal focusing on the pathological basis of all cancers, potential targets for therapy and treatment protocols employed to improve the management of cancer patients. The journal also focuses on the impact of management programs and new therapeutic agents and protocols on

\section{Dovepress}

patient perspectives such as quality of life, adherence and satisfaction. The manuscript management system is completely online and includes a very quick and fair peer-review system, which is all easy to use. Visit http://www.dovepress.com/testimonials.php to read real quotes from published authors. 